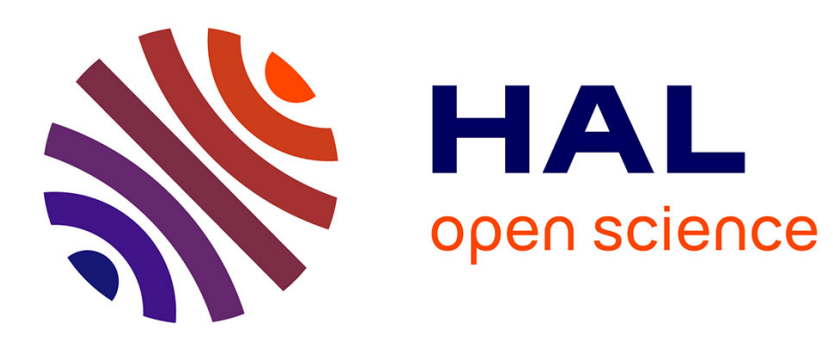

\title{
TOWARDS SAFE-BIM CURRICULA BASED ON THE INTEGRATION OF CYBERSECURITY AND BLOCKCHAINS FEATURES
}

Assam Hammi, Abdelaziz Bouras

\section{- To cite this version:}

Assam Hammi, Abdelaziz Bouras. TOWARDS SAFE-BIM CURRICULA BASED ON THE INTEGRATION OF CYBERSECURITY AND BLOCKCHAINS FEATURES. INTED 2018, Mar 2018, Valencia Spain. hal-01737929

\section{HAL Id: hal-01737929 \\ https://hal.science/hal-01737929}

Submitted on 20 Mar 2018

HAL is a multi-disciplinary open access archive for the deposit and dissemination of scientific research documents, whether they are published or not. The documents may come from teaching and research institutions in France or abroad, or from public or private research centers.
L'archive ouverte pluridisciplinaire HAL, est destinée au dépôt et à la diffusion de documents scientifiques de niveau recherche, publiés ou non, émanant des établissements d'enseignement et de recherche français ou étrangers, des laboratoires publics ou privés. 


\title{
TOWARDS SAFE-BIM CURRICULA BASED ON THE INTEGRATION OF CYBERSECURITY AND BLOCKCHAINS FEATURES
}

\author{
A. Hammi, A. Bouras \\ Qatar University (QATAR)
}

\begin{abstract}
During the last decade, Qatar started an industry diversification strategy based on a knowledge-based economy for the next years. The ambition is to become a global "hub" in some key fields such as new technologies, finance, culture and sporting events. Such a diversification strategy requires massive investments, not only in physical and technical infrastructures, but also in the field of education. For instance, big projects based on the use of advanced new technologies such as BIM (Building Information Modelling) are currently under development in new economic cluster areas in the country, such as QEZ (Qatar Economic Zones), Lusail Smart City, Stadiums for FIFA world cup 2022, etc. This raises a need for new BIM competences, and helps local education institutions such Qatar University to adopt a BIM education strategy in collaboration with these sectors and AEC (Architecture, Engineering, and Construction) industry in general. Such effort intends to cover the lack of skills and equips graduates with specific BIM competency. Globally, BIM education in the country remains soft and partial compared to the current evolution of the AEC industry but also to the risks that are inherent to the BIM adoption. This paper deals with the integration of new information technology patterns such as Cybersecurity and its Blockchains feature in a typical university BIM curricula. Addressing this feature during the BIM collaborative processes, such as the sharing information models developed and maintained across the lifecycle of the building/infrastructure, is becoming tremendous. Such curricula are becoming multidisciplinary by nature, and require the collaboration between not only the different education departments (Architecture, Civil Engineering, Computer Science and Engineering...), but also the local concerned industry. An integrated proposal will be made in this paper, focusing on the specific needs of the Qatari context.
\end{abstract}

Keywords: University-Industry collaboration, Curricula, BIM (Building Information Modelling), Cybersecurity, Blockchains.

\section{INTRODUCTION/CONTEXT}

Building Information Modelling (BIM) comes with its intelligent process based on 3D model and information to give the AEC professionals the tools and ways to design, to construct, and to manage building/infrastructure efficiently. Due to its enormous importance, the UK government is mandating it on all public projects from 2016 in order to benefit from all its advantages, actually they are in level 2 of BIM maturity and they will move to level 3 from here 2025, which is deeper in terms of processes and workflows [1]. According to (Bilal Succar), BIM includes a wide variety of concepts, tools and workflows which need to be learned and applied by industry stakeholders [2]. BIM Education covers the procedures of obtaining the necessary knowledge and the required skills to establish BIM deliverables and meet the clients' requirements. BIM Education as well is the process of learning the sum of theoretical and practical knowledge linking to BIM technologies, workflows and protocols [2]. At this age of Information Technology (IT) revolution, BIM is a new field of technology which is currently posing some challenges to educators in terms of its teaching \& training, which are due to the needed resources for knowledge, skills, finance and time, coupled with its influences on project delivery processes [3]. To ensure the success of an integrated curricula, we have to be motivated to use BIM, this can be reached for industry by the governments and major clients' pressure applied, and the opportunity to improve the profits and the competitiveness [4].

The Section above underlines the relation between BIM and IT while Section 2 of this paper touches some of the main works related to BIM education curricula within the high education institutions at an international level, then Section 3 focuses on the current issues related to the safety needs in BIM process, and highlights some of the possible solutions to tackle those needs. Section 4 refers to some BIM-Blockchains application and finally, Section 5 provides an example of local context and a typical BIM education curricula, based on an interdisciplinary BIM certificate under discussion between teams from three departments at Qatar University (computer science and engineering department, the 
architecture and urban planning and the civil and architectural engineering department: CSE, AUP \& $\mathrm{CAE}$ ) within a national priorities research program (NPRP7-1883-5-289 project).

\section{STATE OF THE ART}

The education sector, especially the HEls, is still lacking to meet the industrial needs in terms of manpower for the construction industry. In terms of a national development, human resources represent a major factor and its quality level depends on the society awareness and understanding; which is also based on the quality of education and training [3]. A complete level of education and in particular technical education is crucial for the design and productive use of new technologies. The ability to adopt and practice new ICT systems largely depends on the capacity of the whole society to be educated and to be able to assimilate and process complex information. Therefore, the development of human resources can best be achieved by a standard and by an education system with technological base [5]. Meanwhile architecture and engineering became as separate jobs from what we called in the past "Master Builder", the education of the AEC disciplines' students have been changed in isolation manner from not only each other but even from the industrials where they have to be in order improve their skills by training and cooperative. (Pressman) affirmed that plenty programs in the academia give students wrong expectation when they finish their studies, which is working as brave solitary designers. But integrated practice necessitates a rethinking of that notion. Academia has to teach beyond how to design and detail, to how to engage with and lead others, and how to collaborate within a professional carrier [6]. In order to build a 3D intelligent model (BIM Model), it is crucial to gather the design team from the beginning unlike the classical practice. They will create a simulation of the real project where the accurate quality of the model is related in direct manner to the information and detail that can be added. This new technology is shaking the professionals' roles, by inventing such jobs that never have been existed, i.e. BIM coordinator, BIM manager...etc. Previously, tutors in higher education institutions have refused to teach new technologies as CAD software programs to students. According to (Macdonald, J.A) [7], the refrain "we're not teaching students to press buttons" is continuously heard by lecturers whom are supposing that BIM is just another CAD software. Nevertheless, they didn't realize that BIM is technological process and it comes with plenty advantages far beyond a simple 3D visualization to facilitate tasks, and to change culture. (Macdonald, J.A) proposed an education typical BIM framework which is called IMAC frame work and which is composed of four stages; Illustration, Manipulation, Application and Collaboration "Fig. 1".

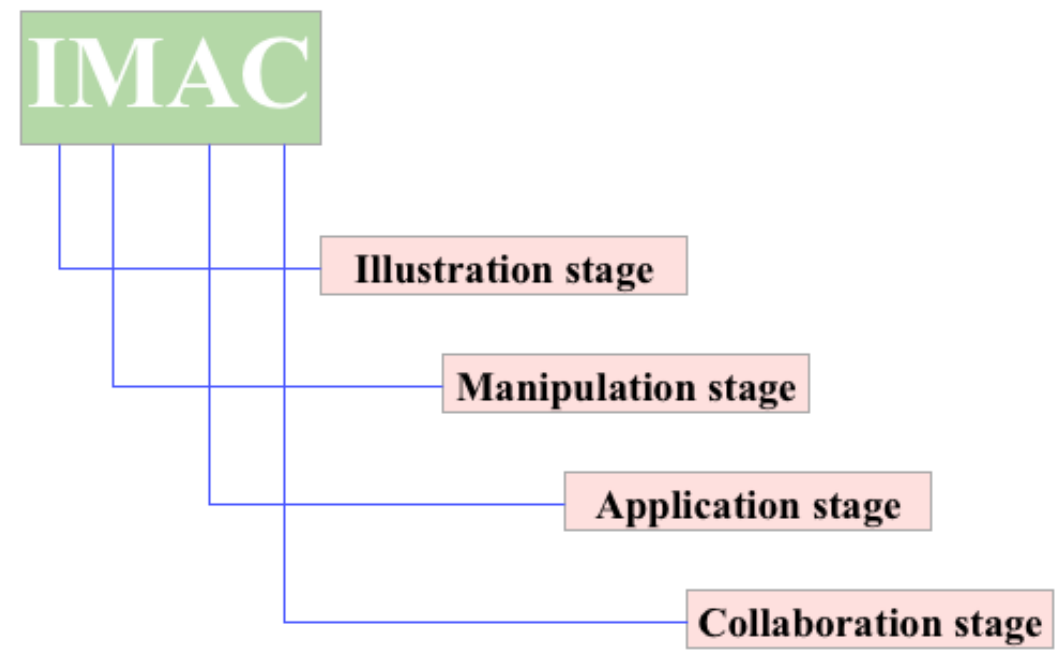

Figure 1. The four stages making up the IMAC framework.

Four stages mentioned above are; (1) Illustration Stage (Knowledge/Comprehension and Receiving/Responding): Here we are talking about a starting stage where the BIM models will be used to illustrate and demonstrate fundamental concepts to students. Those BIM models have a sufficient level of detail that allows lecturers/tutors to highpoint the different elements and objects showing how buildings will be constructed, insulated and waterproofed for example. The students will be taught separately in their proper disciplines. (2) Manipulation Stage (Comprehension/Application and Responding/Valuing): At this phase, students begin interacting with and manipulating the existing BIM models by themselves. They will be prerequisite to make some basic changes and/or create simple 
elements and objects related to their disciplines within the existing BIM models. In parallel, students are developing their coordination working and improving their IT knowledge skills, in addition to enhancing knowledge related to their discipline-specific. (3) Application Stage (Application/Analysis and Valuing/Organizing): Students at this phase, after having acquired the theoretical knowledge, they start to apply it to solve problems related to their disciplines. For architecture students, they start to make the BIM models from sketching and learn how to set these models up for efficient interdisciplinary collaboration. Engineers after that will start to use tools to analyze those models using exports BIM formats. Construction managers will progress and improve the models with the 4D (construction sequencing) and 5D (cost estimation schedules). (4) Collaboration Stage (Synthesis/Evaluation and Characterizing): At this final phase, all the students meet together and start to work on common projects. Real-project issues will be addressed by the students for solving, for the purpose to facilitate the students understanding of the process, they would start working on semifinished BIM models, and then be requested to make some modifications due to "new project information" highlighted. The students will learn the different types of contract that smooth BIM process and protocols, and will continue to learn collaboration and coordination working.

(Zhang, Schmidt and Hui Li) [8] identified priorities for successful curriculum development in Civil Engineering and Management (CEM) utilizing Quality Deployment Function (QFD) as a guideline, in order to respond to the increased need for talent development in the area of BIM in China. Indeed, adopted the QFD framework of industrial engineering education to propose different curriculum planning phases which are described below "Fig. 2".

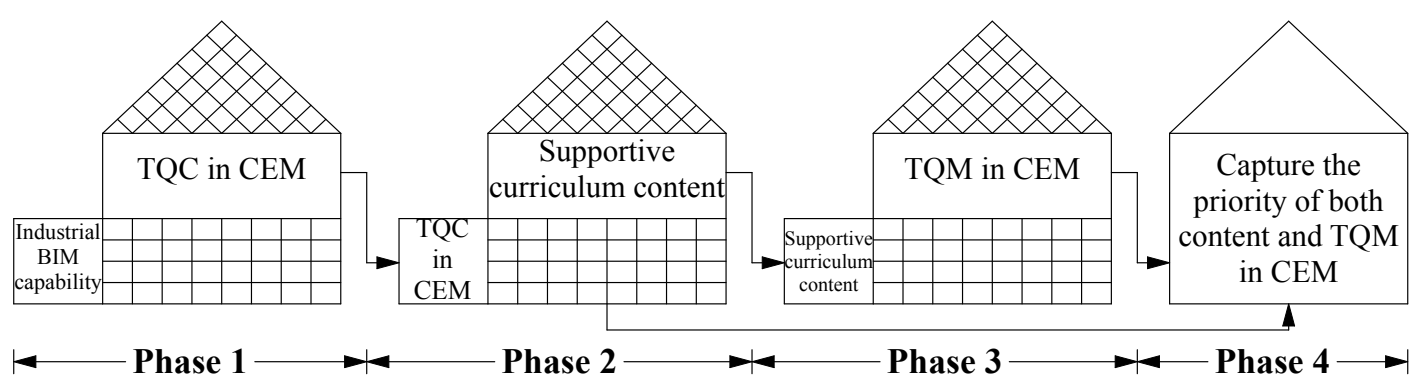

Figure 2. Curriculum planning phases using the Quality Function Deployment (QFD) method.

Phase I is related to the planning curriculum, which highlights the industrial BIM capability for providing critical teaching quality characteristics (TQC), while phase II refers to the design curriculum, where it gives TQC the priority to be included into the supportive curriculum content. In phase III, which refers to the implementation curriculum, Teaching Quality Management (TQM) is attributed to specific curriculum content in Civil Engineering and Management, and finally the phase IV which concerns the priority curriculum is integrating the phases II and III to provide a curriculum content in addition to TQM techniques and modules in order to meet the industrial BIM capability.

(Wu, Tzong-Hann, et al) [9] used Virtual Reality into BIM education curricula, where they designed and implemented two VR types; desktop-based and immersive, which are platforms named the virtual BIM reviewer (VBR) where students use the BIM model to highlight discrepancies by communicating with each other to achieve a solving problem solution. The first VBR was presented into the Sky Classroom courses of 2015 and 2016 for evaluation and was used in 2017, the second VBR was added in 2016 respecting the BIM model review procedure. Three issues were identified to test the remote communication in class i.e. low communicability, passive problem finding, and poor spatial cognition. The VBR functions has been tested in the Sky Classroom course to support seven universities globally collaborating in their course project. Manipulators In the desktop-based VBR, explore the model through the virtual environment using mouse and keyboard, communicating with each other by the chat room and interact through a server that include the BIM model and client data. Users identify the design discrepancies and explore the BIM model utilising the inspection module then they discussed together to highlight those issues by using the communication module. They proceed after for the problem-solving solution using the modification module. Regarding the immersive VR module, it reinforces spatial understanding so it gives them the best design issues identification. Notwithstanding the huge efforts to integrate an efficient BIM education strategy within higher education institutions which intends to cover the lack of skills and equips graduates with specific BIM competency to meet the AEC industry requirements, we observed that it still remains insufficient and 
inadequate due to the new issues and needs related to information security that are coming and should be tackled.

\section{BIM \& SECURITY ISSUES}

Implementation of BIM processes and protocols in many construction projects is definitely a major evolution for the AEC industry sector, all project's data based BIM is shared electronically in a common environment (CDE), using common data environment offers a consistent decisions basis through the lifecycle of a project, from design to destruction. However, some multiple new issues \& risks are facing the BIM adoption related to safety and security, consequently the need to address cyber security awareness and implementation on collaboration systems and coordination processes. Cyber security is beyond than a just technology; it involves people, process and governance issues, and their inter-relationships. As BIM encompasses a complex interaction as well between the same actors, it is crucial that all personnel involved in a BIM project understand the cyber security implications [10]. So, the problematic comes from how to secure the sensitive data and how to manage the pertinent documents between inter-disciplinary teams. From that point of view, we distinguish that there is less trust between different BIM actors compared with the traditional process information with plenty of singed and, stamped and firmly bound pieces of paper. Such a complicated collaboration environment when using BIM involved varied teams from the AEC sector is creating automatically security issues, the fact that industrials do not have the same levels of security understanding and standards. Moreover, the change of the interfacing people and the growth of the supply chain during the project lifecycle make the protection of the information uncontrollable and more complex. Hence, the classical BIM skills became not sufficient to master such issues and we need to complete and compensate by new IT patterns and paradigms. Blockchain technology which is a cybersecurity feature would make the record even more trustworthy in a project common data environment (CDE), the fact that all actions are recorded and visible which make a total transparency. The digital development of construction is going more speedily, after geographic information system (GIS), 3D Printing, BIM and Internet of things (IOT) comes the Blockchain, a new technology which used as fintech concept behind the cryptocurrency such as Bitcoin, Ethereum and all worldwide digital payment system [11]. It allows trust in a business that lacks it. All business done in a blockchain environment is recorded many times in the servers of all those given access, so nobody can break into the record and alter it without it being obvious. The term 'distributed ledger' is sometimes used to describe it, with each block of data solidly linked to all others in a chain of blocks. More broadly speaking, the Blockchain enables a better tracking and administration of contractual documentation which cannot be achieved securely with BIM. Raising operational efficiency by digitalizing the storage of contracts in a secure manner is a big step forward for the construction industry "Fig. 3".

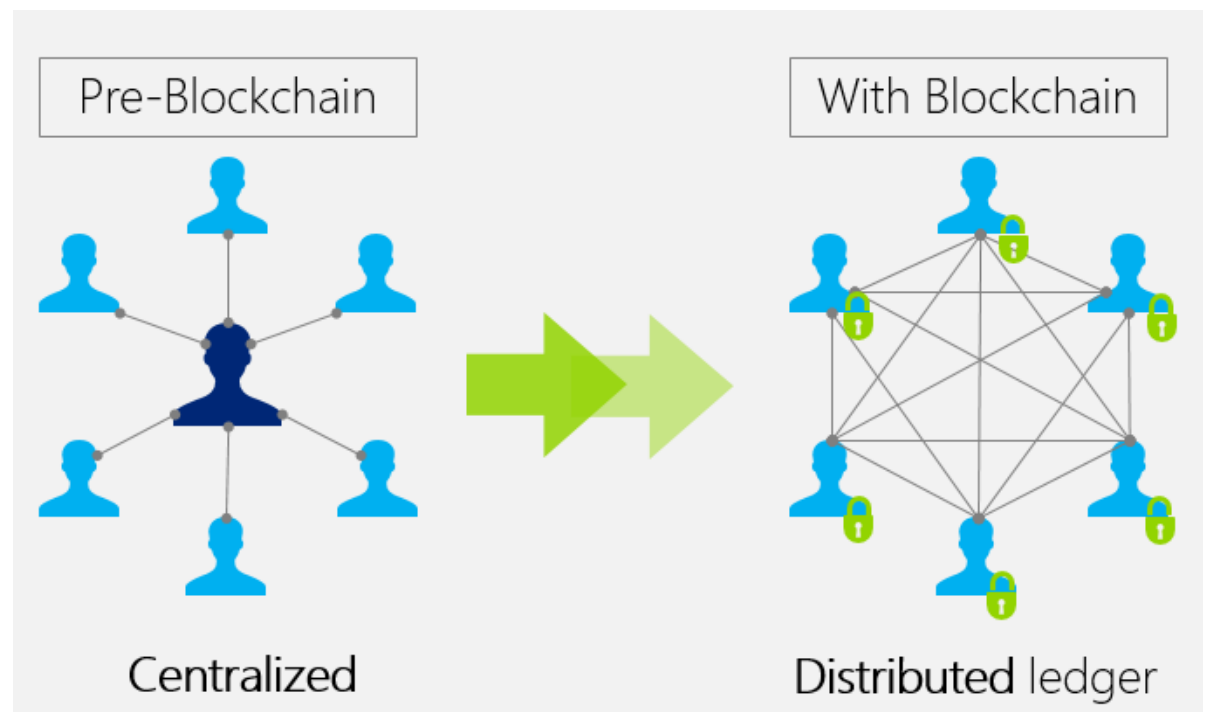

Figure 3. Blockchain technology concept. 


\section{BLOCKCHAIN BASED FRAMEWORKS}

Integrated through BIM processes and protocols, Blockchain is representing a very powerful and promising tool that will make a concrete evolution in the AEC industry sector in the coming years. The implementation of the Blockchain technology is currently used in few domains but differently. For example if we take its application in the finance (Bitcoin, Etherieum,..etc), we realize that the number of transactions, number of participants and size of the data to be managed are huge compared with those components in the BIM Blockchain which are about hundreds of transactions between dozens of users up to a couple of gigabytes each. Here (Turk and Robert) [12] present 4 scenarios where blockchain would be used in a BIM process which are described below:

(1) Chained and very decentralized: in this scenario BIM files are linked into the blockchain which is already integrated across workstations of participants. All versions of all files are preserved and a valid "last" version of each file is maintained via an operating system plugin. (2) Chained and slightly decentralized: due to the big size of the BIM files in the first scenario that exceeds the capacity of individual workstations as the entire Bitcoin does. This scenario comes with the distribution of the blockchain across a few key project partners in order to offer what is called a "wallet software". It appears to the client that a file is local while in fact it would be pulled from the blockchain and cached locally if and when needed. At least one project partner has to host the entire blockchain. (3) Unchained: this scenario is completely different from the previous ones, it does not store the BIM files themselves in the blockchain but only stores their fingerprints. It uses Cloud or Server to store the BIM files. A copy of the blockchain is given to all project partners as poof to access the document management system. However, it would be left to other software to guarantee that all the files mentioned in the blockchain would be preserved somewhere. (4) Blockchain of BIM transactions: in the last scenario, and in order to reach the best performance using Blockchain within BIM transactions, is preferred to integrate blockchain within a server where the size of blockchain can be expected and better managed, as it is larger than the BIM data which is already controllable with current technology. The architecture of a system using Blockchain within BIM transactions is given here under "Fig. 4", (figure enhanced by authors).

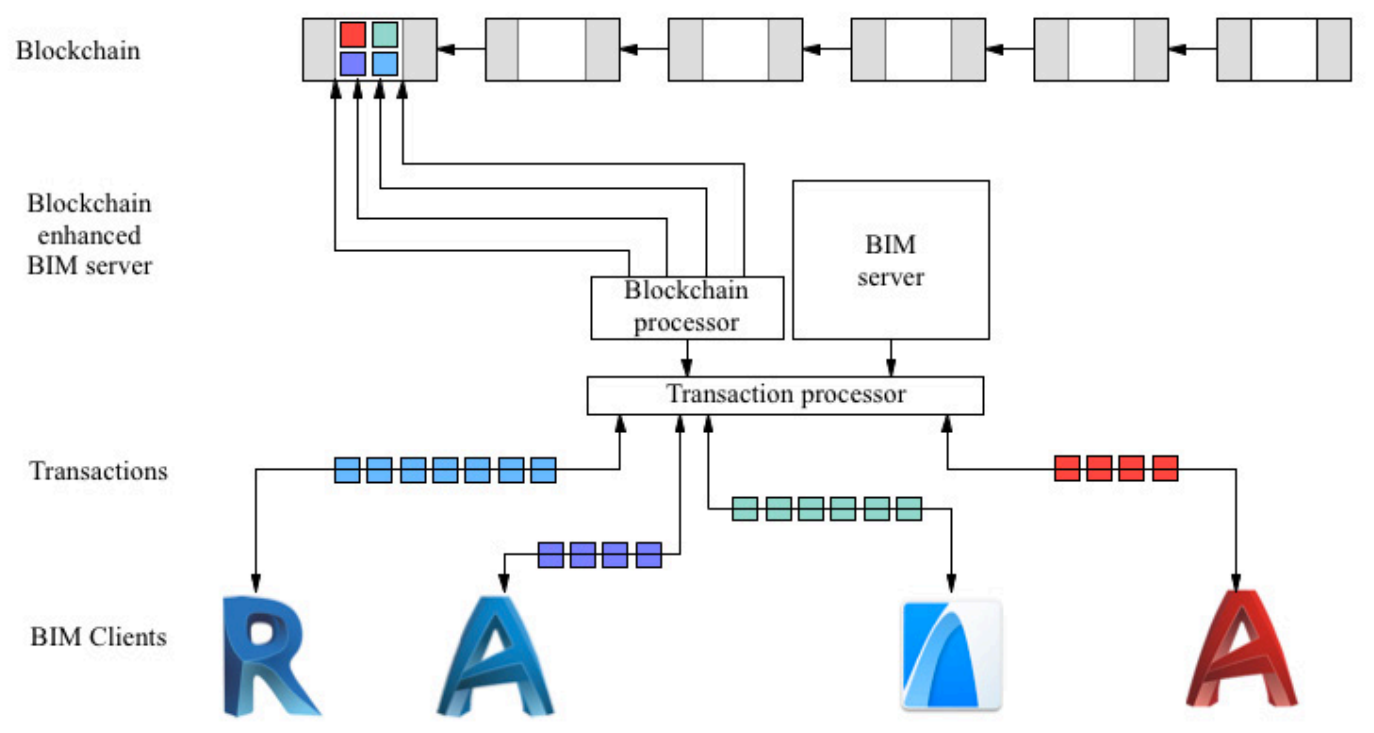

Figure 4. Blockchain within BIM transactions.

Blockchain is also used as a disruptive new technology at the urban level. (Marsal \& Maria) [13] proposed Blockchain4Cities, where the main purpose is to make a coopetition (physical-virtual networking environment). Physical actions executed by humans are registered in the blockchain and controlled in smart contracts. Blockchain4Cities is a Machine to Human system that permits the delivery and the implementation of urban codes. Blockchain-made urban policies codes: urban needs are submitted directly into the blockchain by citizens and the highly demanded ones are transferred to the authorities via consensus mechanism to include them in the official policies draft, and this will cover the real needs of the population unlike the politic agenda. Blockchain-enabled urban planning codes: this is a continuity of the previous step, after the agreement on the policies, their physical transformation is defined by using blockchain where citizens submit master planning's characteristics 
and zooning, this ensure a reasonable distribution of physical involvements. Blockchain-articulated urban regulation codes: this deals with the intangible citizens needs which are not transformable to physical involvements, utilizing the same pervious consensus. Blockchain-facilitated urban standards codes: this comes after all the above steps to design the standards requested based on the citizen's needs. Blockchain4Cities is physical/virtual networking co-operation that allows the deliverance of urban codes using blockchain technology. "Fig. 5", (figure enhanced by authors).

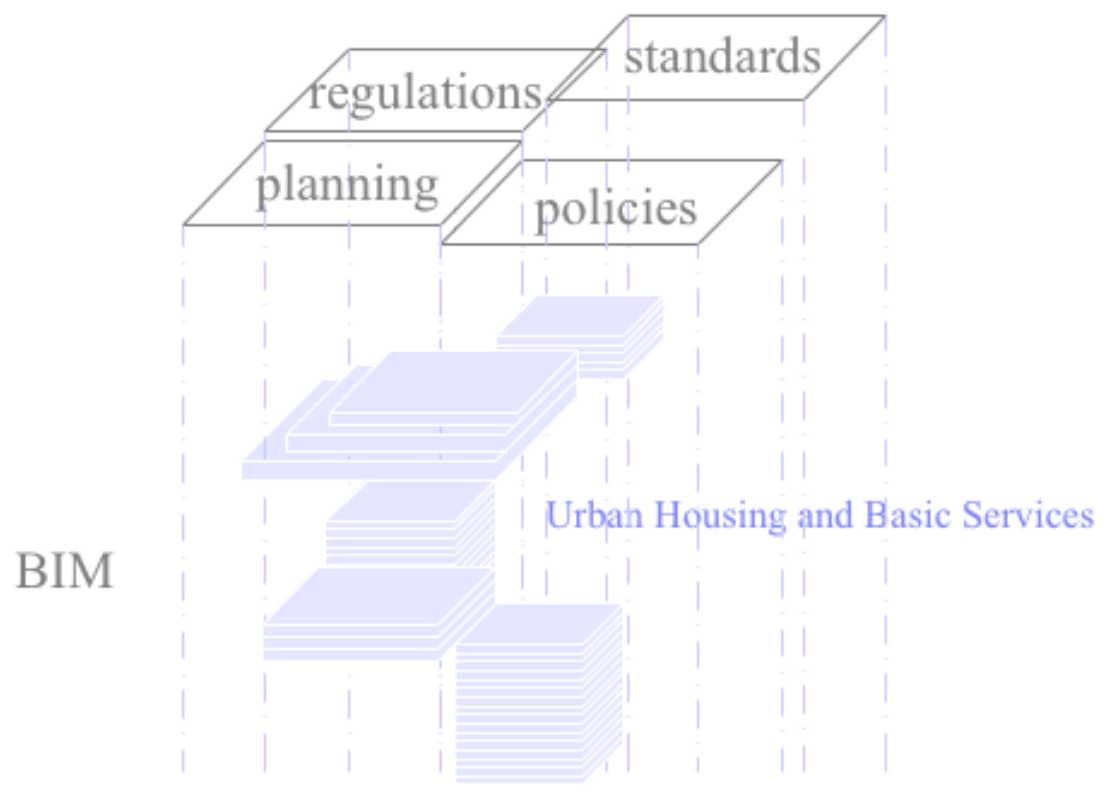

Figure 5. Blockchain4Cities.

While (Mathews, M., Robles, D. \& Bowe, B) [14] highlighted that using Blockchain and BIM will resolve the problem of trust occurred between different stakeholders in the AEC sector. They proposed an \#AECoin cryptocurrency coin which is a collaborative consensus controlled by smart contracts within a blockchain. This helps micro economies to be developed, recording transactions values of the collaboration and providing a method to reward both individual validations and contributions between all the actors by an agreed percentage of coin of that cryptocurrency. This system can change radically the current centralised professional collaboration existed nowadays and gives more trust to project based multidisciplinary collaboration.

\section{AN EXAMPLE OF CURRICULA}

The Qatari government anticipate spending around 160 billion $\$$ on infrastructure and construction projects as Metro System, stadiums, residences and hotels, in preparation of the 2022 FIFA World Cup. (Qatar Construction Summit, 2011). Ensuring the delivering of those projects necessitates a total fulfilment of the requirements and specifications within a specified time. The implementation of BIM process becoms a must. Notwithstanding the increasing use of BIM has been observed in Qatar's AEC sector in the last few years. Indeed, large scale clients such as Qatar Foundation, Qatar Economic Zones, Lusail smart City, Ashghal, Qatar Rail, and the Supreme Committee for Delivery and Legacy have required BIM implementation in their projects. Multiple efforts have been done to elaborate such BIM requirements and regulations for the tenders to restructure the BIM processes and protocols. For example; Lusail smart City is one of the BIM pioneers in Qatar due to its largest BIM projects (buildings/infrastructure) which have been used in many business processes as design and construction, marketing...etc. The Supreme Committee for Delivery \& Legacy intended that the stadiums projects will be constructed fully BIM way, their BIM Implementation Master Plan is a perfect specimen to be followed by any potential consultants/contractors interested in participating in these developments. Ashghal has also implemented BIM requirements on their projects such as expressways and roads, which engendered a lot of benefits for all stakeholders. Such BIM requirements are projected to be adopted soon as standards for highway and tunnelling projects. However, the ability of providing a complete approach regarding BIM processes implementation for the whole AEC sector is still limited. Hence, the necessity to tackle BIM needs becomes crucial and could be achieved by real awareness and BIM education programs. BIM education in Qatar is still 
remaining light if we compare it with the occidental countries such as England or United States where universities came up with a BIM graduate degrees (PhD, Master). However, most of known deposes are mainly focusing on BIM knowledge and deployment. Because of the raising security problems mentioned earlier, it becomes necessary to deal with these issues. This paper proposes a typical educational BIM security curricula in the form of an industry integrated certificate. It has the purpose of improving the level of BIM security competency and understanding among students and BIM experts.

At this moment, a CO-OP option is prepared between 3 Qatar University (CSE, AUP \& CAE) Departments and industry. All learners make alternate stays between two learning venues QU and firms. Two learners' profiles have been proposed "Fig. 6".

\subsection{Profile 1}

The learner is already employed by the firms (employee). Firms have BIM based projects or specific BIM tasks and would like to support the employee who is carrying out of these tasks by a specific certificate. In the proposed certificate, QU will provide the courses and methodological monitoring and the firms provide the professional monitoring and industry projects.

\subsection{Profile 2}

The learner is a young undergraduate (student) who is continuing his/her studies. Firms are interested in hiring him/her as an employee, and interested in funding his/her internship for the duration of the certificate (1 year) within an assigned BIM project. QU and the firms collaborate together on the selection of the students. QU academic staff provide the courses and methodological monitoring and firms provide the professional monitoring, industry projects, etc.

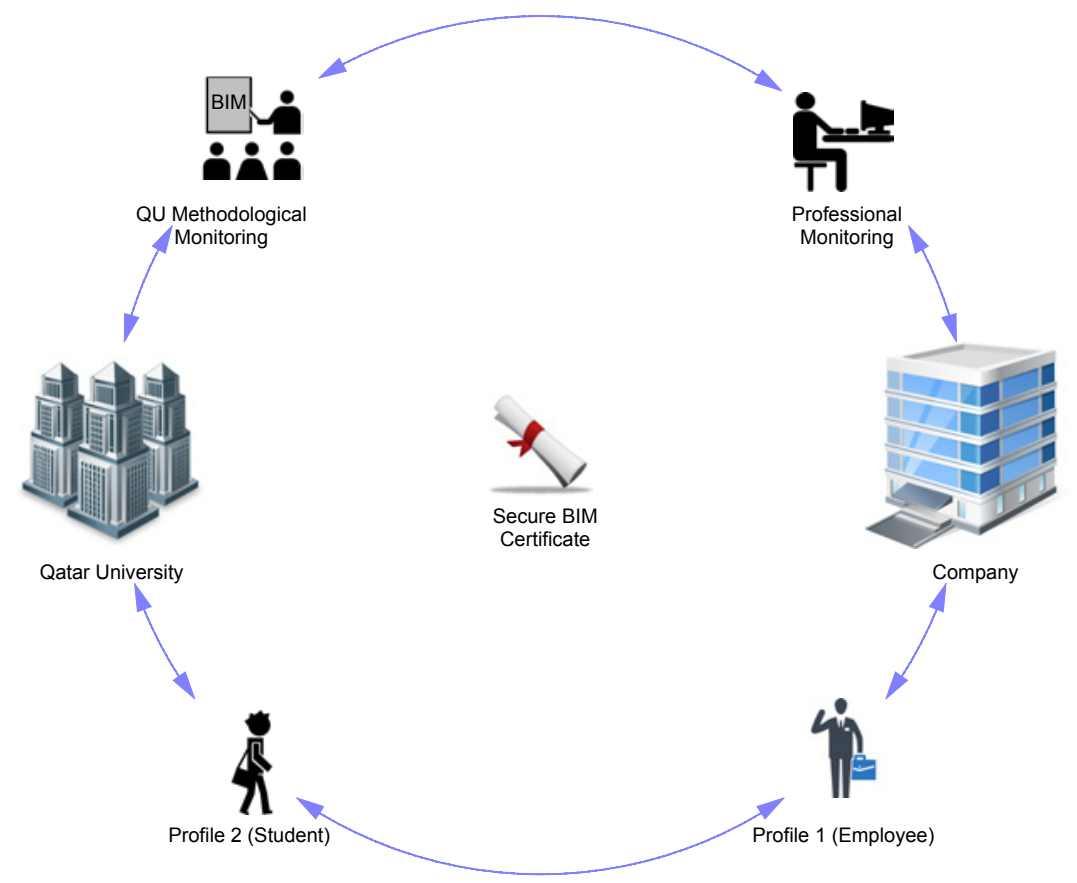

Figure 6. Collaboration Option.

The proposed BIM certificate structure is given hereunder:

- Duration: one year

- Number of courses: Five courses (see table 1).

- Course Project: 1 semester, Industry BIM-based project agreed on with the employer.

- Monitoring/Coaching sessions (QU side): one day every three weeks.

- Participant Engagement: The participant will work full time in the company.

- Other activities: Seminars, workshops, brainstorming, etc.

- Infrastructure: Access to library and university facilities. 
Table 1. BIM Certificate courses

\begin{tabular}{l|c|c}
\hline \hline Course & Course Title & Credit hours \\
\hline Course1 & Applied Research Methodology & 3 Credits \\
\hline Course2 & Programming Concepts & 3 Credits \\
\hline Course3 & Computer Security & 3 Credits \\
\hline Course4 & BIM software & 3 Credits \\
\hline Course5 & Model Based Design software & 3 Credits \\
\hline \hline
\end{tabular}

The new approach requires a closer connection between the CSE/AUP/CAE departments and local partner firms, in order to select projects that meet the real needs of the industry, and that can be as favorable as possible to participant's learning. It also requires a reinforced dialogue between the participant, the firm and the university tutors of the certificate degree.

In addition to these courses, leading experts will be invited on specific topics within seminars and workshops. The certificate contains two courses from CSE department and one course from each AUP/CAE department. The program covers a specific range of courses, such as Programming concepts (i.e. algorithms and problem solving, programming, etc.), Information Security (Cryptography, blockchain fundamentals and application), BIM architecture software (Dynamo for Revit, Python, etc.), Model based design (Scripts, Data sharing for Civil 3D). "Fig. 7".

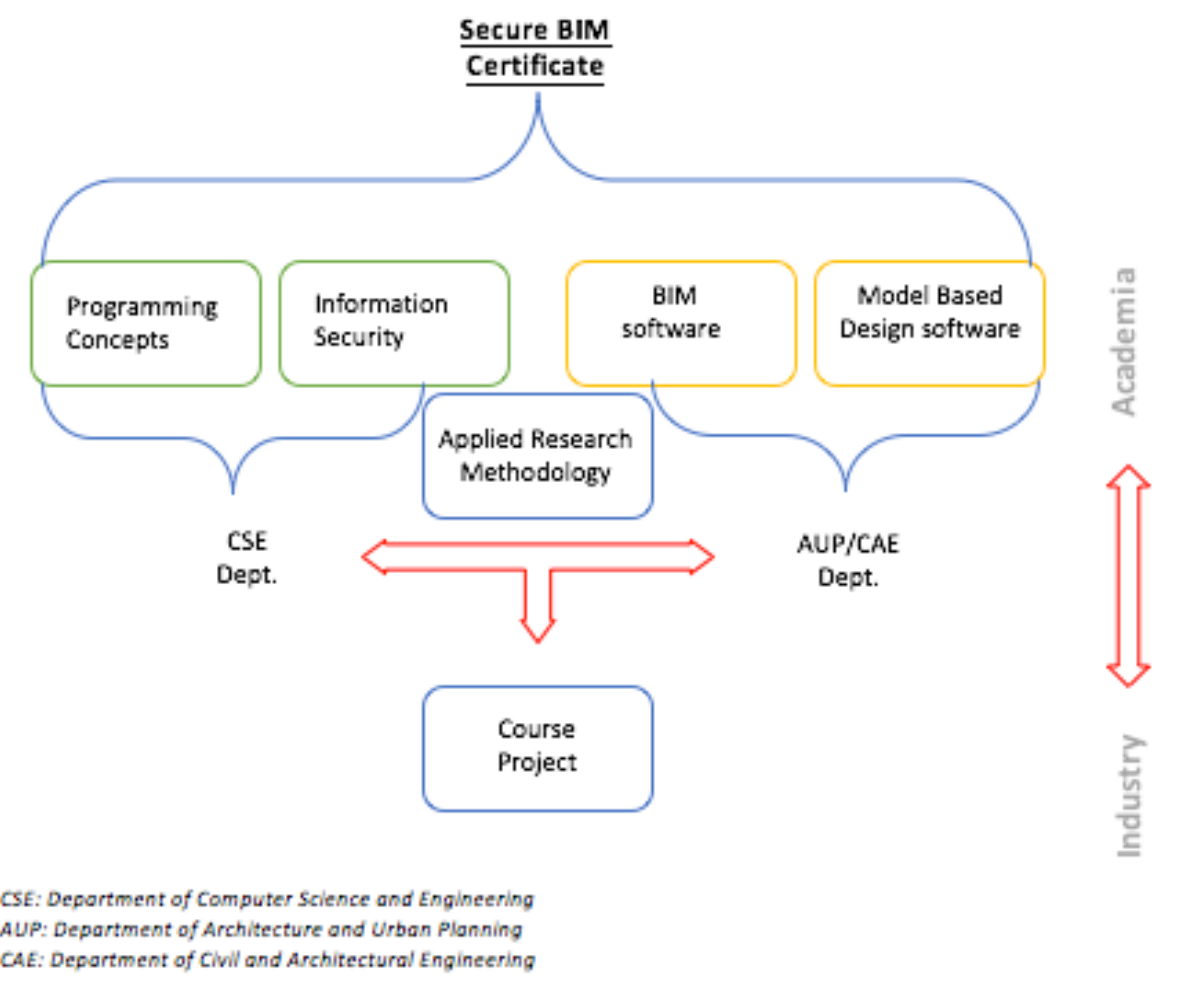

Figure 7. BIM Certificate Cycle.

In order to be more flexible for employees (learners), all courses are scheduled in the end of the afternoon. The study plan requires learners to complete 12 credit hours (4 concentration electives courses selected "Fig. 7". The normal duration of full-time study is one year (two semesters, where one semester is dedicated for the project). The total credit hours have been carefully designed to ensure complementarity and needed skills. 


\section{CONCLUSIONS}

The paper focuses on the current issues related to the safety needs in BIM processes, and highlights new IT paradigm to tackle those needs. A typical BIM education curriculum, based on an interdisciplinary secure BIM certificate, is under discussion between teams from three departments at Qatar University (computer science and engineering department, the architecture and urban planning and the civil and architectural engineering department: CSE, AUP \& CAE), to cover the gaps between industry and academia. An online survey has been designed to better understand the security requirements (https://goo.gl/forms/X7UyxIDgMgHY4U6B3). Workshops, specific brainstorming and seminars with local industry and stakeholders are also scheduled to assess and validate the proposal.

\section{ACKNOWLEDGEMENTS}

This work is part of the NPRP7-1883-5-289 project. The authors would like to express their gratitude to the QNRF (Qatar Foundation) for its support and funding for the project activities.

\section{REFERENCES}

[1] V. Cable, M. Fallon and D. Higgins, "Construction 2025," HM Government: London, UK, p. 80, 2013.

[2] B. Succar, C. Agar, S. Beazley, P. Berkemeier, R. Choy, R. Di Giangregorio, S. Donaghey, C. Linning, J. Macdonald, R. Perey and J. Plume, BIM Education, BIM in Practice, 2012.

[3] B. Y. Yusuf, M. R. Embi and K. N. Ali, "Academic readiness for building information modelling (BIM) integration to Higher Education Institutions (HEIs) in Malaysia," in Research and Innovation in Information Systems (ICRIIS), 2017 International Conference on, 2017.

[4] M. Riel, "Education in the 21st century: Just-in-time learning or learning communities," in Fourth Annual Conference of the Emirates Center for Strategic Studies and Research, Abu Dhabi, 1998.

[5] M. Castells, Information technology, globalization and social development, vol. 114, United Nations Research Institute for Social Development Geneva, 1999.

[6] A. Pressman, "Integrated practice in perspective: A new model for the architectural profession," Architectural Record, vol. 195, pp. 116--+, 2007.

[7] J. A. Macdonald, "A framework for collaborative BIM education across the AEC disciplines," in 37th Annual Conference of Australasian University Building Educators Association (AUBEA), 2012.

[8] J. Zhang, K. Schmidt and H. Li, "BIM and sustainability education: Incorporating instructional needs into curriculum planning in CEM programs accredited by ACCE," Sustainability, vol. 8, p. 525,2016

[9] T.-H. Wu, F. Wu, C.-J. Liang, Y.-F. Li, C.-M. Tseng and S.-C. Kang, "A virtual reality tool for training in global engineering collaboration," Universal Access in the Information Society, pp. 113, 2017.

[10] H. Boyes, "Building Information Modelling (BIM): Addressing the Cyber Security Issues," The Institution of Engineering and Technology: London, UK, 2014.

[11] J. Brito and A. Castillo, Bitcoin: A primer for policymakers, Mercatus Center at George Mason University, 2013.

[12] Turk and R. Klinc, "Potentials of Blockchain Technology for Construction Management," Procedia Engineering, vol. 196, pp. 638-645, 2017.

[13] M.-L. Marsal-Llacuna, "Future living framework: Is blockchain the next enabling network?," Technological Forecasting and Social Change, 2017.

[14] M. Mathews, D. Robles and B. Bowe, "BIM+ Blockchain: A Solution to the Trust Problem in Collaboration?," http://arrow.dit.ie/bescharcon, 2017. 